\title{
Heuristic Algorithms for Multi-Constrained Quality of Service Routing
}

\author{
Xin Yuan
}

\begin{abstract}
Multi-constrained Quality of Service (QoS) routing deals with finding routes that satisfy multiple independent QoS constraints. This problem is NP-hard. In this paper, two heuristics, the limited granularity heuristic and the limited path heuristic, are investigated. Both heuristics extend the Bellman-Ford shortest path algorithm and solve general $k$-constrained QoS routing problems. Analytical and simulation studies are conducted to compare the time/space requirements of the heuristics and the effectiveness of the heuristics in finding paths that satisfy the QoS constraints. The major results of this paper are the followings. For an $N$ nodes and $E$ edges network with $k$ (a small constant) independent QoS constraints, the limited granularity heuristic must maintain a table of size $O\left(|N|^{k-1}\right)$ in each node to be effective, which results in a time complexity of $O\left(|N|^{k}|E|\right)$, while the limited path heuristic can achieve very high performance by maintaining $O\left(|N|^{2} \lg (|N|)\right)$ entries in each node. These results indicate that the limited path heuristic is relatively insensitive to the number of constraints and is superior to the limited granularity heuristic in solving $k$-constrained QoS routing problems when $k>3$.
\end{abstract}

\section{Introduction}

The migration to integrated networks for voice, data and multimedia applications introduces new challenges in supporting predictable communication performance. Multimedia applications require the communication to meet stringent requirement on delay, delay-jitter, cost and/or other quality of service (QoS) metrics. QoS routing, which identifies paths that meet the QoS requirement and selects one that leads to high overall resource efficiency, is the first step toward achieving end-to-end QoS guarantees.

The QoS requirement of a point-to-point connection is specified as a set of constraints, which can be link constraints or path constraints [2]. A link constraint, such as the bandwidth constraint, specifies the restriction on the use of links. For example, the bandwidth constraint requires that each link along the path must be able to support certain bandwidth. A path constraint, such as the delay constraint, specifies the end-to-end QoS requirement for the entire path. For example, the delay constraint requires that the aggregate delay of all links along the path must be less than the delay requirement.

Multi-constrained QoS routing finds a path that satisfies multiple independent path constraints. One example is the delay-cost-constrained routing, i.e., finding a route in the network with bounded end-to-end delay and bounded endto-end cost. We will use the notion $k$-constrained routing

Xin Yuan is with the Department of Computer Science, Florida State University to refer to multi-constrained QoS routing problems with exactly $k$ path constraints. The delay-cost-constrained routing is an example of a $2-$ constrained routing problem. Multiconstrained QoS routing is known to be NP-hard[4, 9]. Previous work $[1,10]$ has focused on developing heuristic algorithms to solve 2-constrained problems. The algorithm in [10] guarantees to find a path that satisfies the QoS constraints if such a path exists. In the worst case, the time complexity of the algorithm may grow exponentially with respect to the network size. Algorithms in [1] find approximate solutions in polynomial time. The general $k$-constrained routing problem receives little attention. In practice, however, effective heuristics to solve general $k$-constrained QoS routing problems, such as the delayjitter-cost-constrained problem, are needed.

This paper considers two polynomial time heuristics, the limited granularity heuristic and the limited path heuristic, that can be applied to the extended Bellman-Ford algorithm to solve $k$-constrained QoS routing problems. The limited granularity heuristic obtains approximate solutions in polynomial time by using finite domains, such as bounded ranges of integer numbers, to approximate the infinite number of values that QoS metrics can take. The limited path heuristic focuses on the cases that occur most frequently in general and solves these cases efficiently and effectively. In this paper, we develop the heuristics to solve the general $k$-constrained QoS routing problems, investigate the performance of the heuristics in solving $k-$ constrained problems, and identify the conditions for the heuristics to be effective. We prove analytically that for an $N$ nodes and $E$ edges network with $k$ independent path constraints ( $k$ is a small constant), the limited granularity heuristic must maintain a table of size $O\left(|N|^{k-1}\right)$ in each node to achieve high probability of finding a path that satisfies the QoS constraints when such a path exists. By maintaining a table of size $O\left(|N|^{k-1}\right)$, the time complexity of the limited granularity heuristic is $O\left(|N|^{k}|E|\right)$. The analysis also shows that the performance of the limited path heuristic is rather insensitive to $k$ and that the limited path heuristic can achieve very high performance by maintaining $O\left(|N|^{2} l g(|N|)\right)$ entries in each node. These results indicate that the limited granularity heuristic is inefficient when $k>3$ since the time/space requirement of the limited granularity heuristic increases drastically when $k$ increases and that the limited path heuristic is more effective than the limited granularity heuristic in solving $k$-constrained QoS routing problems when $k>3$. The simulation study 
further confirms this conclusion.

The rest of the paper is organized as follows. Section 2 discusses the related work. Section 3 describes the multiconstrained QoS routing problem and introduces the extended Bellman-Ford algorithm that can solve this problem. Section 4 studies the limited granularity heuristic for $k$-constrained problems. Section 5 analyzes the limited path heuristic. Section 6 presents the simulation study. Section 7 concludes the paper.

\section{Related Work}

Much work has been done in QoS routing recently, an extensive survey can be found in [2]. Among the proposed QoS routing schemes, the ones that deal with multiconstrained QoS routing are more related to the work in this paper. In [8], a distributed algorithm was proposed to find paths that satisfy the end-to-end delay constraint while minimizing the cost. Although this algorithm considers two path constraints, it does not solve the 2 -constrained problem because the cost metric is not bounded. Ma [6] showed that when the weighted fair queuing algorithm is used, the metrics of delay, delay-jitter and buffer space are not independent and all of them become functions of the bandwidth. Orda [7] proposed the quantization of QoS metrics for efficient QOS routing in networks with a ratebased scheduler at each router. Although the idea of quantization of QoS metrics is similar to the limited granularity heuristic, it was proposed in [7] to improve the performance of a polynomial time QoS routing algorithm that solves the bandwidth-delay bound problem. Jaffe [4] proposed a distributed algorithm that solves 2 -constrained problems with a time complexity of $O\left(|N|^{5} b \log (|N| b)\right)$, where $b$ is the largest value of the weights. This algorithm is pseudopolynomial in that the execution time depends on the value of the weights (not just the size of the network). Widyono [10] proposed an algorithm that performs exhaustive search on the QoS paths in exponential time. Chen [1] and Korkmaz [5] proposed heuristic algorithms that effectively solves 2-constrained problems. This research differs from the previous work in that it studies heuristic algorithms that efficiently solve the general $k$-constrained QoS routing problem. Some of the results for 2-constrained QoS routing [1] are special cases of the results in this paper.

\section{$3 \quad$ Background}

\subsection{Assumptions and notations}

The network is modeled as a directed graph $G(N, E)$, where $N$ is the set of nodes representing routers and $E$ is the set of edges representing links that connect the routers. Each edge $e=u \rightarrow v$ is associated with $k$ independent weights, $w_{1}(e), w_{2}(e), \ldots, w_{k}(e)$, where $w_{l}(e)$ is a positive real number $\left(w_{l}(e) \in R^{+}\right.$and $\left.w_{l}(e)>0\right)$ for all $1 \leq l \leq k$. The notation $w(e)=w(u \rightarrow v)=\left(w_{1}(e), w_{2}(e), \ldots, w_{k}(e)\right)$ is used to represent the weights of a link. It is assumed that all the constraints are path constraints and that the weight functions are additive [9], that is, the weight of a path is equal to the summation of the weights of all edges on the path. Thus, for a path $p=v_{0} \rightarrow v_{1} \rightarrow v_{2} \rightarrow \ldots \rightarrow v_{n}$, $w_{l}(p)=\sum_{i=1}^{n} w_{l}\left(v_{i-1} \rightarrow v_{i}\right)$. Notation $w(p) \leq w(q)$ denotes $w_{l}(p) \leq w_{l}(q)$ for all $1 \leq l \leq k$. Other relational operators $<,=,>, \geq$ and arithmetic operators + , - on the weight vectors are defined similarly. Let a path $p=v_{0} \rightarrow v_{1} \rightarrow v_{2} \rightarrow \ldots \rightarrow v_{n}$ and a link $e=v_{n} \rightarrow v_{n+1}$. The notation $p+e$ or $p+\left\{v_{n} \rightarrow v_{n+1}\right\}$ denotes the path $v_{0} \rightarrow v_{1} \rightarrow v_{2} \rightarrow \ldots \rightarrow v_{n} \rightarrow v_{n+1}$. This paper considers centralized algorithms and assumes that the global network state information is known.

Given a set $S$, the notation $|S|$ denotes the size of the set $S$. We will use the following notations: binary logarithm function $l g(n)=\log _{2}(n)$, natural logarithm function $\ln (n)=\log _{e}(n)$, power of the logarithm function $l g^{k}(n)=$ $(\lg (n))^{k}$, and factorial function $n !=n *(n-1) * \ldots * 1$. We define $0 !=1$.

\subsection{Multi-Constrained QoS Routing}

Definition 1: Given a directed graph $G(N, E)$, a source node $s r c$, a destination $d s t, k \geq 2$ weight functions $w_{1}$ : $E \rightarrow R^{+}, w_{2}: E \rightarrow R^{+}, \ldots, w_{k}: E \rightarrow R^{+}$, and $k$ constants $c_{1}, c_{2}, \ldots \quad c_{k}$ represented by a vector $c=\left(c_{1}, c_{2}, \ldots, c_{k}\right)$, multi-constrained $Q o S$ routing is to find a path $p$ from $s r c$ to $d s t$ such that $w(p) \leq c$, that is, $w_{1}(p) \leq c_{1}, w_{2}(p) \leq c_{2}$, $\ldots, w_{k}(p) \leq c_{k}$.

We will call a multi-constrained routing problem with $k$ weight functions a $k$-constrained problem. Since the number of weight functions in a network is small, we will assume that $k$ is a small constant.

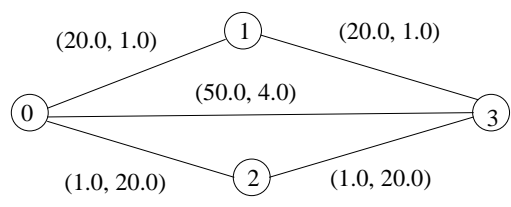

Figure 1: Optimal QoS paths

Definition 2: Given a directed graph $G(N, E)$ with $k \geq 2$ weight functions $w_{1}: E \rightarrow R^{+}, w_{2}: E \rightarrow R^{+}, \ldots, w_{k}:$ $E \rightarrow R^{+}$, a path $p=s r c \rightarrow v_{1} \rightarrow v_{2} \rightarrow \ldots \rightarrow d s t$ is said to be an optimal QoS path from src to dst if there does not exist another path $q$ from $s r c$ to $d s t$ such that $w(q)<w(p)$.

When $k=1$, the optimal QoS path is the same as the shortest path. When $k>1$, however, there can be multiple optimal QoS paths between two nodes. For example, in Figure 1, both path $p_{1}=0 \rightarrow 1 \rightarrow 3\left(w\left(p_{1}\right)=(40.0,2.0)\right)$ and path $p_{2}=0 \rightarrow 2 \rightarrow 3\left(w\left(p_{2}\right)=(2.0,40.0)\right)$ are optimal QoS paths from node 0 to node 3. Path $p_{3}=0 \rightarrow 3$ is not an optimal QoS path since $w\left(p_{3}\right)=(50.0,4.0)>w\left(p_{1}\right)$. Optimal QoS paths are interesting because each optimal QoS path can potentially satisfy particular QoS constraints that no other path can satisfy. On the other hand, when there exists a path that satisfies the QoS requirement, there always exists an optimal QoS path that satisfies the same 


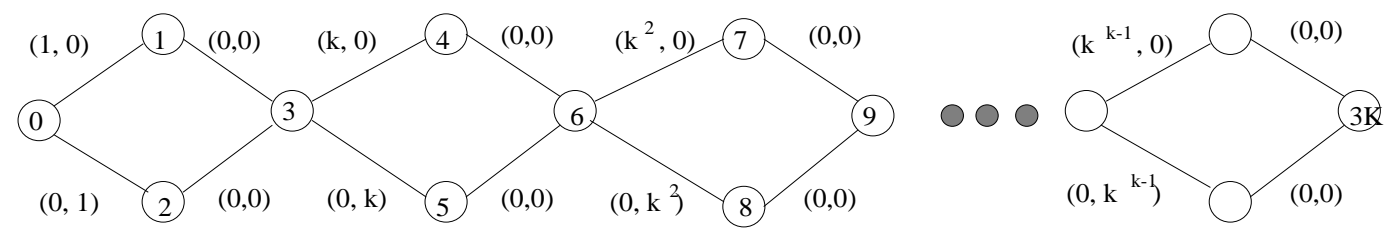

Figure 2: The number of optimal QoS paths between two nodes

$\operatorname{RELAX}(\mathrm{u}, \mathrm{v}, \mathrm{w})$

(1) For each $w(p)$ in $\operatorname{PATH}(\mathrm{u})$

(2) $\quad$ flag $=1$

(3) For each $w(q)$ in $\operatorname{Path}(\mathrm{v})$

(4) if $(w(p)+w(u, v) \geq w(q))$ then

(5) $\quad$ flag $=0$

(6) if $(w(p)+w(u, v)<w(q))$ then

(7) remove $w(q)$ from $P A T H(v)$

(8) if $($ flag $=1)$ then

(9) $\quad$ add $w(p)+w(u, v)$ to $P A T H(v)$

$\operatorname{BELLMAN-FORD}(G, w, c, s r c, d s t)$

(1) For $i=0$ to $|N(G)|-1$

(2) $\operatorname{PATH}(i)=\phi$

(3) $P A T H($ src $)=\{\overrightarrow{0}\}$

(4) For $i=1$ to $|N(G)|-1$

(5) For each edge $(u, v) \in E(G)$

(6) $\operatorname{RELAX}(\mathrm{u}, \mathrm{v}, \mathrm{w})$

(7) For each $w(p)$ in $P A T H(d s t)$

(8) if $(w(p)<c)$ then return "yes"

(9) return "no"

Figure 3: The extended Bellman-Ford algorithm (EBFA) for multi-constrained QoS routing problems

QoS requirement. Thus, a QoS routing algorithm can guarantee finding a path that satisfies the QoS constraints when such a path exists if the algorithm considers all optimal QoS paths. Notice that the number of optimal QoS paths can be exponential with respect to the network size as shown in Figure 2. In Figure 2, the number of optimal QoS paths from node $s r c=0$ to node $d s t=3 k$ is equal to $2^{k}$ because from each node $3 i$ where $0 \leq i<k$, taking the link $3 i \rightarrow 3 i+1$ or $3 i \rightarrow 3 i+2$ will result in different optimal QoS paths.

\subsection{Extended Bellman-Ford Algorithm}

Since the heuristics that we consider are variations of the extended Bellman-Ford algorithm, we will describe a version of the extended Bellman-Ford algorithm in this section for the completeness of the paper. Figure 3 shows the algorithm, which is a variation of the Constrained BellmanFord algorithm in [10]. For simplicity, the algorithm only checks whether there exists a path that satisfies the QoS constraints. The algorithm can easily be modified to find the exact path. We will call the algorithm EBFA.

$E B F A$ extends the original Bellman-Ford shortest path algorithm [3] by having each node $u$ to maintain a set $P A T H(u)$ that records all optimal QoS paths found so far from src to $u$. The first three lines in the main routine (BELLMAN_FORD) initialize the variables. Lines (4) to (6) perform the relax operations. After the relax operations all optimal QoS paths from node $s r c$ to node $d s t$ are stored in the set PATH(dst). Lines (7) and (8) check whether there exists an optimal QoS path that satisfies the QoS constraints. The RELAX $(u, v, w)$ operation is a little more complicated since all the elements in $\operatorname{PATH}(u)$ and $P A T H(v)$ must be considered. For each element $w(p)$ in $P A T H(u)$, line (4) in the RELAX routine checks whether there exists an old path $q$ from $s r c$ to $v$ that is better than path $p+(u \rightarrow v)$. If such a path exists, then $p+(u \rightarrow v)$ is not an optimal QoS path. Line (6) checks whether path $p+(u \rightarrow v)$ is better than any old path from src to $v$. If such an old path $q$ exists, then path $q$ is not an optimal QoS path and is removed from the set $P A T H(v)$. Line (8) adds the newly found optimal QoS path to $P A T H(v)$.

$E B F A$ guarantees to find a path that satisfies the QoS constraints when such a path exists by recording all optimal QoS paths in each node. Given a network $G(N, E)$, the algorithm executes the RELAX operation $O(|N||E|)$ times. The time and space needed to execute $R E L A X(u, v, w)$ depends on the sizes of $P A T H(u)$ and $P A T H(v)$, which are the number of optimal QoS paths from node $s r c$ to nodes $u$ and $v$ respectively. Since the number of optimal QoS paths from src to $u$ or $v$ can be exponential with respect to $|N|$ and $|E|$, the time and space requirement of $E B F A$ may also grow exponentially. Thus, heuristics must be developed to reduce the time and space complexity.

The idea of both the limited granularity heuristic and the limited path heuristic is to limit the number of optimal QoS paths maintained in each node, that is, the size of $P A T H$, to reduce the time and space complexity of the RELAX operation. By limiting the size of $P A T H$, each node is not able to record all optimal QoS paths from the source and the heuristics can only find approximate solutions. Thus, the challenge of the heuristics is how to limit the size of $P A T H$ in each node while maintaining the effectiveness in finding paths that satisfy QoS constraints. In the next few sections, we will discuss two different methods to limit the size of $P A T H$ and study their performance when solving general $k$-constrained QoS routing problems. 
$\operatorname{RELAX}(\mathrm{u}, \mathrm{v}, \mathrm{w})$

(1) for each $d^{v}\left[i_{2}, i_{3}, \ldots, i_{k}\right]$

(2) Here, $1 \leq i_{2} \leq X_{2}, \ldots, 1 \leq i_{k} \leq X_{k}$

(3) Let $d^{v}[\vec{i}]=d^{v}\left[i_{2}, i_{3}, \ldots, i_{k}\right]$

(4) Let $j_{l}$ be the largest $j_{l}$ such that $r_{j_{l}}<r_{i_{l}}-w_{l}(u, v), 2 \leq l \leq k$

(5) Let $d^{v}[\vec{j}]=d^{v}\left[j_{2}, j_{3}, \ldots, j_{k}\right]$

(6) if $\left(j_{l} \geq 1\right.$, for all $\left.2 \leq l \leq k\right)$ then

(7) $\quad$ if $\left(d^{v}[\vec{i}]>d^{u}[\vec{j}]+w_{1}(u, v)\right)$ then

(8) $\quad d^{v}[\vec{i}]=d^{u}[\vec{j}]+w_{1}(u, v)$

Limited_Granularity_Heuristic $(G, w, c, s r c, d s t)$

(1) For $i=0$ to $|N(G)|-1$

(2) For each $d^{i}\left[i_{2}, i_{3}, \ldots, i_{k}\right]$

(3) Here, $1 \leq i_{2} \leq X_{2}, \ldots, 1 \leq i_{k} \leq X_{k}$

(4) if $(i=s r c)$ then $d^{s r c}\left[i_{2}, i_{3}, \ldots, i_{k}\right]=0$

(5) $\quad$ else $d^{i}\left[i_{2}, i_{3}, \ldots i_{k}\right]=\infty$

(6) For $i=1$ to $|N(G)|-1$

(7) For each edge $(u, v) \in E(G)$

(8) $\operatorname{RELAX}(\mathrm{u}, \mathrm{v}, \mathrm{w})$

(9) if $\left(d^{d s t}\left[X_{2}, X_{3}, \ldots, X_{k}\right]<c_{1}\right)$ then return TRUE

(10)return FALSE

Figure 4: The limited granularity heuristic for $k_{-}^{-}$ constrained routing problems

\section{The limited granularity heuristic}

When all QoS metrics except one take bounded integer values, the multi-constrained QoS routing problem is solvable in polynomial time. The idea of the limited granularity heuristic is to use bounded finite ranges to approximate QoS metrics, which reduces the original NP-hard problem to a simpler problem that can be solved in polynomial time. This algorithm is a generalization of the algorithms in [1]. To solve the $k$-constrained problem defined in Section 3.2, the limited granularity heuristic approximates $k-1$ metrics with $k-1$ bounded finite ranges. Let $w_{2}, \ldots, w_{k}$ be the $k-1$ metrics to be approximated, that is, for $2 \leq i \leq k$, the range $\left(0, c_{i}\right]$ is mapped into $X_{i}$ elements, $r_{1}^{i}, r_{2}^{i}, \ldots, r_{X_{i}}^{i}$, where $0<r_{1}^{i}<r_{2}^{i}<\ldots<r_{X_{i}}^{i}=c_{i}$. The $w_{i}(e) \in\left(0, c_{i}\right]$ is approximated by $r_{j}^{i}$ if and only if $r_{j-1}^{i}<w_{i}(e) \leq r_{j}^{i}$. In the rest of the section, we will use the notation $a w_{i}(p), 2 \leq i \leq k$, to denote the approximated $w_{i}(p)$ in the bounded finite domain $\left\{r_{1}^{i}, r_{2}^{i}, \ldots, r_{X_{i}}^{i}\right\}$.

Figure 4 shows the limited granularity heuristic that solves $k$-constrained problems. In this heuristic, each node $u$ maintains a table $d^{u}\left[1: X_{2}, 1: X_{3}, \ldots, 1: X_{k}\right]$. An entry $d^{u}\left[i_{2}, i_{3}, \ldots, i_{k}\right]$ in the table records the path that has the smallest $w_{1}$ weight among all paths $p$ from the source to node $u$ that satisfy $w_{2}(p) \leq r_{i_{2}}^{2}, w_{3}(p) \leq r_{i_{3}}^{3}, \ldots$, $w_{k}(p) \leq r_{i_{k}}^{k}$. In the $\operatorname{RELAX}(u, v, w)$ operation, to compute $d^{v}\left[i_{2}, i_{3}, \ldots, i_{k}\right]$, only $d^{u}\left[j_{2}, j_{3}, \ldots, j_{k}\right]$ where $j_{l}$ is the largest $j_{l}$ such that $r_{j_{l}}^{l} \leq r_{i_{l}}^{l}-w_{l}(u, v)$, for $2 \leq l \leq k$, needs to be considered. The RELAX routine has a time complexity of $O\left(X_{2} X_{3} \ldots X_{k}\right)$. Notice that the approxima- tion of the weights is carried out implicitly in the $R E L A X$ operation. For example, if, for each path $p$ from $s r c$ to $d s t$, there exists an $i, 2 \leq i \leq k$, such that $a w_{i}\left(p=s r c \rightarrow v_{0} \rightarrow\right.$ $\left.v_{1} \rightarrow \ldots \rightarrow d s t\right)>c_{i}$, then $d^{d s t}\left[X_{2}, X_{3}, \ldots, X_{k}\right]=\infty$ at the end of the algorithm after all RELAX operations are done.

Let $X=X_{2} X_{3} \ldots X_{k}$ be the size of the table maintained in each node. By limiting the granularity of the QoS metrics, the limited granularity heuristic has a time complexity of $O(X|N||E|)$. The most important issue of this heuristic is to determine the relation between the size of the table (which, in turn, determines the time complexity of the heuristic) and the effectiveness of the heuristic in finding paths that satisfy the $k$ QoS constraints. The following lemmas attempt to answer this question.

Lemma 1: In order for the limited granularity heuristic to find any path of length $L$ that satisfies the QoS constraints, the size of the table in each node must be at least $L^{k-1}$. That is, $X=X_{2} X_{3} \ldots X_{k} \geq L^{k-1}$.

Proof: Assuming $X=X_{2} X_{3} \ldots X_{k}<L^{k-1}$, there exists an $i, 2 \leq i \leq k$, such that $X_{i}<L$. Let $p=v_{0} \rightarrow v_{1} \rightarrow$ $v_{2} \rightarrow \ldots \rightarrow v_{L}$ be a path that satisfies the QoS constraints $w(p) \leq c$. Let the range $\left(0, c_{i}\right]$ be approximated by $X_{i}$ discrete elements, $r_{1}^{i}, r_{2}^{i}, \ldots, r_{X_{i}}^{i}$, where $0<r_{1}^{i}<r_{2}^{i}<\ldots<$ $r_{X_{i}}^{i}=c_{i}$.

Let $p(n)$ denote the path $v_{0} \rightarrow v_{1} \rightarrow v_{2} \rightarrow \ldots \rightarrow v_{n}$. By induction, it can be shown that $a w_{i}(p(n)) \geq r_{n}^{i}$. Base case, when $n=1$, since $r_{1}^{i}$ is the smallest value that can be used to approximate, $a w_{i}\left(v_{0} \rightarrow v_{1}\right) \geq r_{1}^{i}$. Assuming that $a w_{i}(p(n-1)) \geq r_{n-1}^{i}, a w_{i}(p(n))=a w_{i}(p(n-1))+$ $a w_{i}\left(v_{n-1} \rightarrow v_{n}\right) \geq r_{n-1}^{i}+w_{i}\left(v_{n-1} \rightarrow v_{n}\right)>r_{n-1}^{i} \geq r_{n}^{i}$. Thus, $a w_{i}\left(p\left(X_{i}\right)\right) \geq r_{X}^{i}=c_{i}$. When $L>X_{i}, a w_{i}(p(L))>$ $c_{i}$. That is, the approximation value for the $w_{i}(p)$ weight is larger than $c_{i}$. Thus, the heuristic does not recognize the path as a path that satisfies $w(p) \leq c$.

Lemma 1 shows that in order for the limited granularity heuristic to be effective in finding paths of length $L$ that satisfy $k$ independent path constraints, the number of entries in each node should be at least $L^{k-1}$. For an $N$-node network, paths can potentially be of length $N$. Thus, the limited granularity heuristic should at least maintain a table of size $O\left(|N|^{k-1}\right)$ in each node to be effective. This result indicates that the limited granularity heuristic is quite sensitive to the number of constraints, $k$. Notice that this lemma does not make any assumptions about the values of $X_{2}, \ldots, X_{k}$ and the values of $r_{j}^{i}$, where $2 \leq i \leq k$ and $1 \leq j \leq X_{i}$. Thus, it applies to all variations of the limited granularity heuristic.

Lemma 2: Let $n$ be a constant, $X_{2}=X_{3}=\ldots=X_{k}=$ $n L$ so that $X=X_{2} X_{3} \ldots X_{k}=n^{k-1} L^{k-1}$. For all $2 \leq$ $i \leq k$, let the range $\left(0, c_{i}\right]$ be approximated with equally spaced values $\left\{r_{1}^{i}=\frac{c_{i}}{X_{i}}, r_{2}^{i}=\frac{c_{i}}{X_{i}} * 2, \ldots, r_{X_{i}}^{i}=c_{i}\right\}$. The limited granularity heuristic guarantees finding a path $q$ that satisfies $w(q) \leq c$ if there exists a path $p$ of length $L$ that satisfies

$w_{1}(p) \leq c_{1}$ and $w_{i}(p) \leq c_{i}-\frac{c_{i}}{n}$ for all $2 \leq i \leq k$.

Proof: Consider the approximation of any $i$ th weight of path $p, 2 \leq i \leq k$, 


$$
\begin{aligned}
a w_{i}(p) & =\sum_{(u \rightarrow v) \text { on } p} a w_{i}(u \rightarrow v) \\
& <\sum_{(u \rightarrow v) \text { on } p}\left(w_{i}(u \rightarrow v)+\frac{c_{i}}{X_{i}}\right) \\
& =\sum_{(u \rightarrow v) \text { on } p} w_{i}(u \rightarrow v)+\sum_{(u \rightarrow v) \text { on } p} \frac{c_{i}}{X_{i}} \\
& \leq c_{i}-\frac{c_{i}}{n}+\frac{L}{X_{i}} * c_{i}=c_{i}
\end{aligned}
$$

Thus, the approximation of all $w_{i}$ weights, $2 \leq i \leq k$, will satisfy the condition $w_{i}(p) \leq c_{i}$. Since the heuristic does not approximate the $w_{1}$ weight, the heuristic can guarantee finding that path $p$ satisfies $w(p) \leq c$.

Lemma 2 shows that when each node maintains a table of size $n^{k-1}|N|^{k-1}=O\left(|N|^{k-1}\right)$ and when $n$ is a reasonably large constant, the limited granularity heuristic can find most of the paths that satisfy the QoS constraints. Furthermore, by maintaining a table of size $n^{k-1} N^{k-1}$, the heuristic guarantees finding a solution when there exists a path whose QoS metrics are better than $\left(1-\frac{1}{n}\right) * \vec{c}$, where $\vec{c}$ is the required QoS metrics of the connection. This guarantee will be called finding an $\left(1-\frac{1}{n}\right)$-approximate solution. For example, if $n=100$, the heuristic guarantees finding a path $p$ that satisfies $w(p) \leq c$ when there exists a path $q$ that satisfies $w(q) \leq 0.99 * c$, that is, it guarantees finding a 0.99-approximate solution.

\section{The limited path heuristic}

The limited path heuristic ensures the worst case polynomial time complexity by maintaining a limited number of optimal QoS paths, say $X$ optimal QoS paths, in each node. Here, $X$ corresponds to the size of the table maintained in each node in the limited granularity heuristic. The limited path heuristic is basically the same as the extended Bellman-Ford algorithm in Figure 3 except that before a path is inserted into $P A T H$, the size of $P A T H$ is checked. When $P A T H$ already contains $X$ elements, the new path will not be inserted. By limiting the size of $P A T H$ to $X$, the time complexity of the RELAX operation is reduced to $O\left(X^{2}\right)$. The time complexity of the heuristic is $O\left(X^{2}|N||E|\right)$.

We must choose the value $X$ carefully for the heuristic to be both efficient and effective. If $X$ is sufficiently large such that each node actually records all optimal QoS paths, the heuristic is as effective as EBFA. However, large $X$ results in an inefficient heuristic in terms of the time/space complexity. In this section, we will show that for any small constant $k$ and a randomly generated network, the limited path heuristic can solve general $k$-constrained problems with very high probability when $X=O\left(|N|^{2} l g(|N|)\right)$. This result indicates that unlike the limited granularity heuristic, the limited path heuristic is insensitive to the number of QoS constraints in the network.

Let us assume that the weights of the links in a graph are randomly generated and are independent of one another. For a set $S$ of $|S|$ paths of the same length, we derive the probability $\operatorname{prob}_{i}$ that set $S$ contains $i$ optimal QoS paths. We then show that when $X=O\left(|N|^{2} \lg (|N|)\right), \sum_{i=1}^{X}$ prob $_{i}$ is very large (or $\sum_{i=X+1}^{|S|} \operatorname{prob}_{i}$ is very small), which indicates that when each node maintains $O\left(|N|^{2} l g(|N|)\right)$ entries, the limited path heuristic will have very high prob-

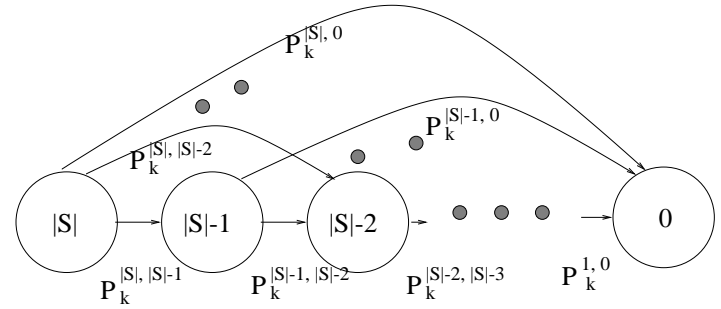

Figure 5: The Markov Chain

ability to record all optimal QoS paths in each node and thus, will have very high probability to find the QoS paths when such paths exist.

We use the following process to derive the probability $\operatorname{prob}_{i}$ that the set $S$ contains $i$ optimal QoS paths. First, the path, $p$, that has the smallest $w_{1}$ weight is chosen from $S$. The path $p$ is an optimal QoS path because $w_{1}(p)$ is the smallest among all the paths. All paths whose $w_{j}$ weights, $2 \leq j \leq k$, are larger than $w_{j}(p)$ are not optimal QoS paths. Let the set $T$ include all such non-optimal QoS paths. The set $S-T$ contains all paths $q$ where there exists at least one $j, 2 \leq j \leq k$, such that $w_{j}(q)<w_{j}(p)$. Thus, a path in the set $S-T$ may potentially be an optimal QoS path. The process is then repeated on the set $S-T$. If $S$ contains $m$ optimal QoS paths, the process can be repeated $m$ times.

Let us use the notion $P_{k}^{i, j}$ to represent the probability of the remaining set size equal to $j$ when the process is applied to a set of $i$ paths and the number of QoS metrics is $k$. We will always assume $0 \leq j \leq i-1$, when the notion $P_{k}^{i, j}$ is used. The process can be modeled as a Markov process as shown in Figure 5. The Markov chain contains $|S|+1$ states, each state $i$ in the Markov chain represents a set of $i$ paths. The transition matrix for the Markov chain is

$$
A_{k}=\left(\begin{array}{cclcc}
0 & 0 & & 0 & 0 \\
P_{k}^{1,0} & 0 & \cdots & 0 & 0 \\
P_{k}^{2,0} & P_{k}^{2,1} & \cdots & 0 & 0 \\
\vdots & \vdots & \cdots & \vdots & \vdots \\
P_{k}^{|S|-1,0} & P_{k}^{|S|-1,1} & \cdots & 0 & 0 \\
P_{k}^{|S|, 0} & P_{k}^{|S|, 1} & \cdots & P_{k}^{|S|,|S|-1} & 0
\end{array}\right)
$$

Let us define $A_{k}^{1}=A_{k}$ and $A_{k}^{m}=A_{k}^{m-1} A_{k}$ for $m>1$. $A_{k}^{m}(i, j)$ represents the probability of the state transferring from node $i$ to node $j$ in exactly $m$ steps. For example, $A_{k}^{1}(|S|, 0)$ represents the probability of a set of size $|S|$ became empty after one optimal QoS path is chosen. $A_{k}^{m}(|S|, 0)$ is the probability that the set of size $|S|$ becomes empty after selecting exactly $m$ optimal QoS paths, that is, $A_{k}^{m}(|S|, 0)$ is the probability that the set $\mathrm{S}$ contains exactly $m$ optimal QoS paths. Our goal is to determine the value $X$ such that $\sum_{m=X}^{|S|} A_{k}^{m}(|S|, 0)$ is very small.

The summation form for $A_{k}^{m}(i, j)$, where $i>j$, are given next. Note that $A_{k}^{m}(i, j)=0$, when $i \leq j$. By definition, we have

$$
A_{k}^{1}(i, j)=P_{k}^{i, j}
$$




$$
\begin{aligned}
A_{k}^{2}(i, j)= & \sum_{n=0}^{|S|} A_{k}(i, n) * A_{k}(n, j) \\
= & \sum_{n=1}^{i=1} P_{k}^{i, n} * P_{k}^{n, j} \\
A_{k}^{3}(i, j)= & \sum_{n_{1}=0}^{|S|} A_{k}\left(i, n_{1}\right) * A_{k}^{2}\left(n_{1}, j\right) \\
= & \sum_{n_{1}=j+2}^{i=1} P_{k}^{i, n_{1}} \sum_{n_{2}=j+1}^{n_{1}-1} P_{k}^{n_{1}, n_{2}} * P_{k}^{n_{2}, j} \\
\ldots \ldots & \\
A_{k}^{m}(i, j)= & \sum_{n_{1}=j+m-1}^{i-1} P_{k}^{i, n_{1}} \sum^{n_{m}} \ldots \\
& \sum_{n_{m-1}=j+1}^{n_{m-2}-1} P_{k}^{n_{m-2}, n_{m-1}} * P_{k}^{n_{m-1}, j}
\end{aligned}
$$

We will derive the numerical bounds for $A_{k}^{m}(i, j)$ in the rest of the section. Let us first consider the 2 -constrained QoS routing problem. When $k=2$, each link has two weights $w_{1}$ and $w_{2}$. In the path selection process, we choose from the set of $i$ paths a path whose $w_{1}$ weight is the smallest. Since $w_{2}$ and $w_{1}$ weights are independent and the length of the paths are the same, the probability of the size of the remaining set may be $0,1, \ldots, i-1$, each with probability $\frac{1}{i}$, since $w_{2}(p)$ can be ranked $1, \ldots ., i$ among the $i$ paths in the set with equal probability $\frac{1}{i}, P_{2}^{i, j}=\frac{1}{i}$. Hence,

$$
A_{2}=\left(\begin{array}{cccccc}
0 & 0 & 0 & & 0 & 0 \\
\frac{1}{1} & 0 & 0 & \cdots & 0 & 0 \\
\frac{1}{2} & \frac{1}{2} & 0 & \cdots & 0 & 0 \\
\vdots & \vdots & \vdots & \cdots & \vdots & \vdots \\
\frac{1}{|S|} & \frac{1}{|S|} & \frac{1}{|S|} & \cdots & \frac{1}{|S|} & 0
\end{array}\right)
$$

By manipulating the matrix $A_{2}$, we have: $A_{2}^{1}(|S|, 0)=$ $\frac{1}{|S|}, A_{2}^{2}(|S|, 0)=\frac{1}{|S|}\left(\frac{1}{|S|-1}+\frac{1}{|S|-2} \ldots+\frac{1}{1}\right)=\frac{1}{|S|} \sum_{i=1}^{|S|-1} \frac{1}{i}$ and for $m \geq 2$,

$$
A_{2}^{m}(|S|, 0)=\frac{1}{|S|} \sum_{i_{1}=m-1}^{|S|-1} \frac{1}{i_{1}} \sum_{i_{2}=m-2}^{i_{1}-1} \frac{1}{i_{2}} \sum_{i_{3}=m-3}^{i_{2}-1} \ldots \sum_{i_{m-1}=1}^{i_{m-2}-1} \frac{1}{i_{m-1}} .
$$

Lemma 3: For $m \geq 2, A_{2}^{m}(|S|, 0) \leq \frac{(2 \ln (|S|))^{m-1}}{|S| *(m-1) !}$.

Proof: See the Appendix.

Theorem 1: Given an $N$ node graph with 2 independent constraints, the limited path heuristic has very high probability to record all optimal QoS paths and thus, has very high probability to find a path that satisfies the QoS constraints when one exists, when each node maintains $O\left(|N|^{2} \lg (|N|)\right)$ paths.

Proof: From Lemma $3, A_{2}^{m}(|S|, 0) \leq \frac{(2 \ln (|S|))^{m-1}}{|S|(m-1) !}$. Using the formula $n ! \geq \sqrt{2 \pi n}\left(\frac{n}{e}\right)^{n}$ from [3], when $m>2 e^{2} \ln (|S|)+$ 1 ,

$$
\begin{aligned}
A_{2}^{m}(|S|, 0) & \leq \frac{(2 \ln (|S|))^{m-1}}{|S|(m-1) !} \\
& \leq \frac{1}{|S|}\left(\frac{2 e \ln (|S|)}{m-1}\right)^{m-1} \\
& \leq \frac{1}{|S|}\left(\frac{1}{e}\right)^{2 e^{2}} \ln (|S|) \\
& \leq \frac{1}{|S|^{2 e^{2}+1}}
\end{aligned}
$$

The number of paths of length $L$ between any two nodes in the graph is at most $R=|N|^{L}$. The probability that there exists no more than $i$ optimal QoS paths among the $R=|N|^{L}$ paths is $p=1-\sum_{m=i+1}^{R} A_{k}^{m}(R, 0)$. When $i>$ $2 e^{2} \ln (R)+1, p=1-\sum_{m=i+1}^{R} A_{k}^{m}(R, 0) \geq 1-\sum_{k=i+1}^{R} \frac{1}{R^{2 e^{2}+1}}$ $\geq 1-\frac{1}{R^{2 e^{2}}}$. Thus, when each node maintains $2 e^{2} \ln \left(|N|^{L}\right)+$ $1=2 e^{2} \operatorname{Llg}(|N|)+1$ paths, the probability that the node can record all optimal QoS paths of length $L$ is very high, $1-\frac{1}{R^{2 e^{2}}}$. For example, if $R=30$, the probability is more than $1-\frac{1}{R^{2 e^{2}}}>99.99999 \%$. In an $N$ node graph, the length of any QoS path is between 1 and $N$. Thus, maintaining $\sum_{L=1}^{|N|} 2 c e^{2} \operatorname{Lln}(|N|)+1=O\left(|N|^{2} l g(|N|)\right)$ paths in each node will give very high probability to record all optimal QoS paths in a node. Thus, the limited granularity heuristic has very high probability to find a path that satisfies the QoS constraints when such a path exists, when each node maintains $O\left(|N|^{2} \lg (|N|)\right)$ paths.

Next, we will derive the formula for computing the general $P_{k}^{i, j}$ and prove that maintaining $O\left(|N|^{2} l g(|N|)\right)$ paths enables the heuristic to solve the general $k$-constrained problem with very high probability. Lemma 4 shows how to compute $P_{k}^{i, j}$.

Lemma 4: $P_{k}^{i, j}=\frac{1}{i} \sum_{l=0}^{j} P_{k-1}^{i-l, j-l}$.

Proof: Let $S$ be the set of $i$ paths. Let $p$ be the path with the smallest $w_{1}(p)$. Consider $w_{2}(p)$, since the weights are randomly generated and are independent, $w_{2}(p)$ can be ranked $1,2, \ldots$, i among all the paths with equal probability $\frac{1}{i}$. In other words, the probability that there are $l, 0 \leq l \leq$ $i-1$, paths whose $w_{2}$ weights are smaller than $w_{2}(p)$ is $\frac{1}{i}$. When $l=0$, all the $i-1$ paths are potential candidates to be considered for the rest $k-2$ weights in the remaining set. In this case, the probability that the remaining set size equal to $j$ is equivalent to the case to choose from $i$ paths the path with the smallest $w_{1}$ weight and the remaining set size is equal to $j$ with $k-1$ weights. Thus, the probability is $P_{k-1}^{i, j}$. When $l=1$, there exists one path $q$ where $w_{2}(q)<$ $w_{2}(p)$, thus, path $q$ belongs to the remaining set. In this case, the probability that the remaining set size equal to $j$ is equivalent to the case to choose from $i-1$ paths (all paths but path $q$ ) the path with the smallest $w_{1}$ weight and the remaining set size is equal to $j-1$ with $k-1$ weights (since path $q$ is already in the remaining set by considering $w_{2}$ ). Thus, the probability is $P_{k-1}^{i-1, j-1}$. Similar arguments apply for all cases from $l=0$ to $l=j$. When $l>j$, there will be at least $l$ paths in the remaining set, thus, the probability that the remaining set size equal to $j$ is 0 . Combining all these cases, we obtain

$$
\begin{aligned}
P_{k}^{i, j} & =\frac{1}{i} P_{k-1}^{i, j}+\frac{1}{i} P_{k-1}^{i-1, j-1}+\ldots+\frac{1}{i} P_{k-1}^{i-j, 0} \\
& =\frac{1}{i} \sum_{l=0}^{j} P_{k-1}^{i-l, j-l} \cdot \square
\end{aligned}
$$

Lemmas 5, 6, and 7 summarize some property of $P_{k}^{i, j}$ and $A_{k}$. See the appendix for the proofs of these lemmas.

Lemma 5: $P_{k}^{i, j}>P_{k}^{i+1, j}$.

Lemma 6: For $k \geq 3$ and $0 \leq j \leq|S|$,

$$
\sum_{i=0}^{|S|} A_{k}(i, j)=\sum_{i=j+1}^{|S|} P_{k}^{i, j}<2
$$

Lemma 7: $P_{k}^{i, j} \leq \frac{1}{i}\left(\frac{1}{i}+\frac{1}{i-1}+\ldots+\frac{1}{i-j}\right)^{k-2}$.

Lemmas Lemmas 8, 9 and 10 are mathematic formulae to be used later. See the appendix for the proofs of these lemmas.

Lemma 8: For a constant $k$, there exists a constant $c$ such that $\sum_{i=1}^{\infty} \frac{1}{2^{i}} i^{k} \leq c$.

Lemma 9: For a constant $\mathrm{k}$ and $1 \leq j \leq i-1$, there exists a constant $c$ such that

$$
\sum_{n=j+1}^{i-1}\left(\frac{1}{i}+\frac{1}{i-1}+\ldots \frac{1}{i-n}\right)^{k}\left(\frac{1}{n}+\ldots+\frac{1}{n-j}\right)^{k} \leq c * i .
$$


Lemma 10: Let $0 \leq j<\frac{i}{2}$, there exists a constant $c$ such that

$$
\sum_{n=j+1}^{i-1} P_{k}^{n, j}\left(\frac{1}{i}+\ldots+\frac{1}{i-n}\right)^{m} \leq c .
$$

The next lemma describes the relation between $A_{2}(i, j)$ and $A_{k}^{2}(i, j)$.

Lemma 11: There exists a constant $c$ such that $A_{k}^{2}(i, j) \leq$ $c * A_{2}(i, j)$.

Proof: Consider the following three cases:

- Case 1: $j \geq i-1$. In this case, $A_{k}^{2}(i, j)=0 \leq A_{2}(i, j)$.

- Case 2: $\frac{i}{2} \leq j \leq i-2$. In this case,

$A_{k}^{2}(i, j)=\sum_{n=j+1}^{i-1} P_{k}^{i, n} * P_{k}^{n, j}$

$\leq \sum_{n=j+1}^{i-1} \frac{1}{i}\left(\frac{1}{i}+\frac{1}{i-1}+\ldots+\frac{1}{i-n}\right)^{k} * \frac{1}{n}\left(\frac{1}{n}+\ldots+\frac{1}{n-j}\right)^{k}$

$\leq \frac{2}{i^{2}} \sum_{n=j+1}^{i-1}\left(\frac{1}{i}+\frac{1}{i-1}+\ldots+\frac{1}{i-n}\right)^{k}\left(\frac{1}{n}+\frac{1}{n-1}+\ldots+\frac{1}{n-j}\right)^{k}$

$\leq \frac{2 c_{1}}{i}=2 c_{1} A_{2}(i, j) / *$ applying Lemma $9 * /$

- Case 3: $0 \leq j \leq \frac{i}{2}-1$.

$$
\begin{aligned}
A_{k}^{2}(i, j) & =\sum_{n=j+1}^{i-1} P_{k}^{i, n} * P_{k}^{n, j} \\
& \leq \sum_{n=j+1}^{i-1} P_{k}^{n, j} \frac{1}{i}\left(\frac{1}{i}+\frac{1}{i-1}+\ldots+\frac{1}{i-n}\right)^{k} \\
& \leq \frac{1}{i} \sum_{n=j+1}^{i-1} P_{k}^{n, j}\left(\frac{1}{i}+\frac{1}{i-1}+\ldots+\frac{1}{i-n}\right)^{k} \\
& \leq \frac{c_{2}}{i}=c_{2} A_{2}(i, j) / * \text { applying Lemma } 10 * /
\end{aligned}
$$

Thus, there exists a constant $c=\max \left(2 c_{1}, c_{2}, 1\right)$ such that $A_{k}^{2}(i, j) \leq c * A_{2}(i, j)$.

Theorem 2: Given an $N$ node graph with $k$ independent constraints, the limited path heuristic has very high probability to record all optimal QoS paths and thus, has very high probability to find a path that satisfies the QoS constraints when one exists, when each node maintains $O\left(|N|^{2} l g(|N|)\right)$ paths.

Proof: From Lemma 3, we have $A_{2}^{m}(|S|, 0) \leq \frac{(2 \ln (|S|))^{m-1}}{|S|(m-1) !}$. From Lemma 11, we have $A_{k}^{2}(i, j) \leq c A_{2}(i, j)$, where $c$ is a constant. Hence $A_{k}^{m}(|S|, 0) \leq c^{\frac{m}{2}} A_{2}^{\frac{m}{2}}(|S|, 0) \leq \frac{c(2 c l n(|S|))^{\frac{m}{2}}-1}{|S|\left(\frac{m}{2}-1\right) !}$

Following the similar arguments as the proof of Theorem 1, it can be shown that the limited granularity heuristic has very high probability to find a path that satisfies the QoS constraints when such a path exists, when each node maintains $O\left(|N|^{2} l g(|N|)\right)$ paths.

Theorem 2 establishes that the performance of the limited path heuristic is not as sensitive to the number of QoS constraints as the limited granularity heuristic. Thus, the limited path heuristic provides better performance when $k>3$. Given that the global network state information is inherently imprecise, in practice, using an algorithm that can precisely solve the $k$-constrained routing problem may not have much advantage over the limited path heuristic that can solve the $k$-constrained routing problem with very high probability.

The proof of Theorem 2 assumes that paths of different lengths are of the same probability to be the optimal QoS paths. However, when the weights in a graph are randomly generated with uniform distribution, the paths of shorter length are more likely to be the optimal QoS paths. In addition, the probability used in the proof of the theorem is extremely high. In practice, we do not need such high probability for the heuristic to be effective. A tighter upper bound for the number of optimal QoS paths to be

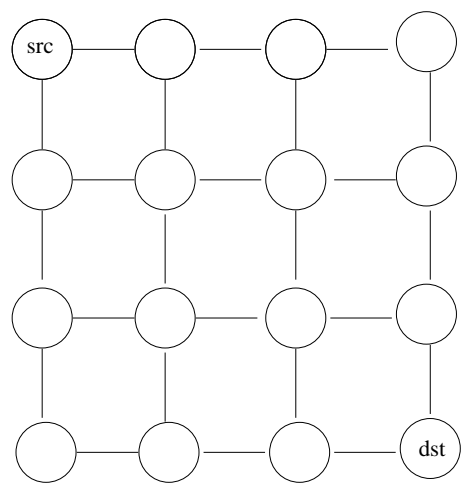

(a) A $4 \times 4$ mesh

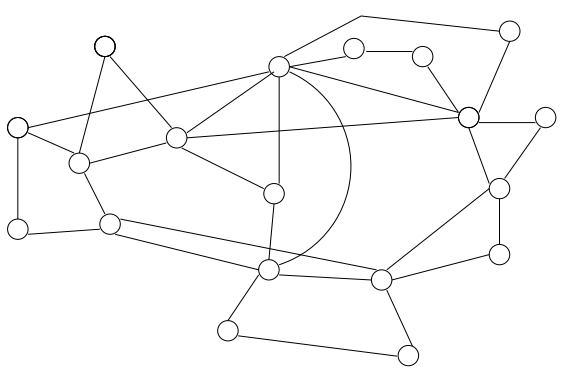

(b) MCI backbone

Figure 6: The network topologies

maintained in each node for the limited path heuristic to be effective may be obtained by considering these factors. However, the formal derivation of a tighter upper bound can be complicated. In the next section, we examine the two heuristics through simulation study.

\section{Simulation Study}

The goal of the simulation experiments is to compare the performance of the heuristics for real world network topologies and to study the impact of constants in the asymptotic bounds we derived. Two topologies, the mesh topology shown in Figure 6 (a) and the MCI backbone topology shown in Figure 6 (b), are used in the studies. In the simulation, the $w_{i}$ weight of each link is randomly generated in the range of $(0.0,10.0 * i)$, for $1 \leq i \leq k$. Since the performance of the two heuristics is closely related to the length of the paths, when the mesh topology is used, we choose to establish connections between the source and the destination that are the farthest apart as shown in Figure 6 (a). When the MCI backbone topology is used, connections are between randomly generated sources and destinations.

We compare the two heuristics with the exhaustive algorithm, $E B F A$, that guarantees finding a path that satisfies the QoS constraints if such a path exists. Two concepts, the existence percentage and the competitive ratio, are used 
to describe the simulation results. The existence percentage, which indicates how difficult the paths that satisfy the QoS constraints can be found, is defined as the ratio of the total number of requests satisfied using the exhaustive algorithm and the total number of requests generated. The competitive ratio, which indicates how well a heuristic algorithm performs, is defined as the ratio of the number of requests satisfied using a heuristic algorithm and the number of requests satisfied using the exhaustive algorithm. By definition, both the existence percentage and the competitive ratio are in the range of $[0.0,1.0]$.

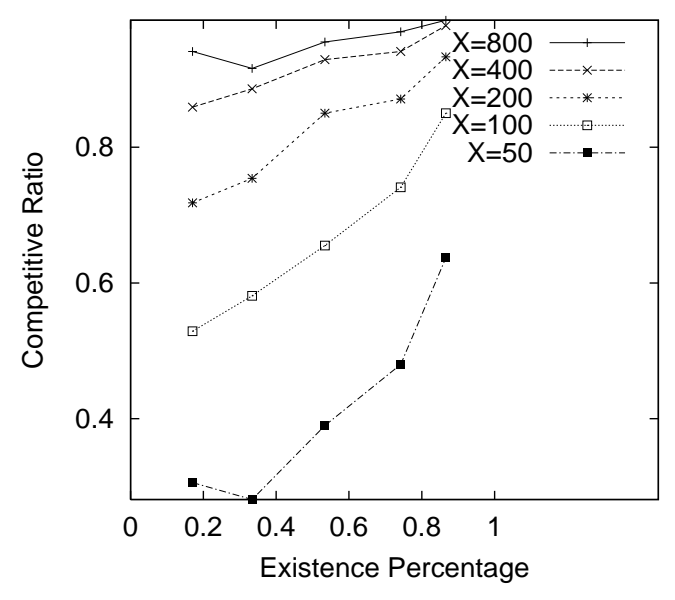

(a) Limited granularity heuristic

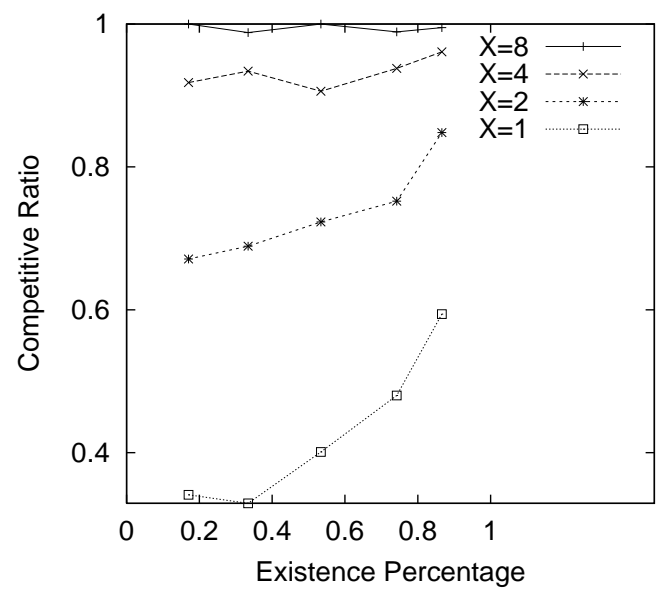

(b) Limited path heuristic

Figure 7: 2-constrained problems on $8 \times 8$ meshes

Figure 7 shows the performance of the two heuristics for $8 \times 8$ meshes with 2 QoS constraints. In both figures, the $\mathrm{x}$-axis represents the existence percentage and the $\mathrm{y}-$ axis represents the competitive ratio. Different curves are for different values of $X$ in the two heuristics. The data for each point in the figure are obtained by running the two heuristics and the exhaustive algorithm using requests

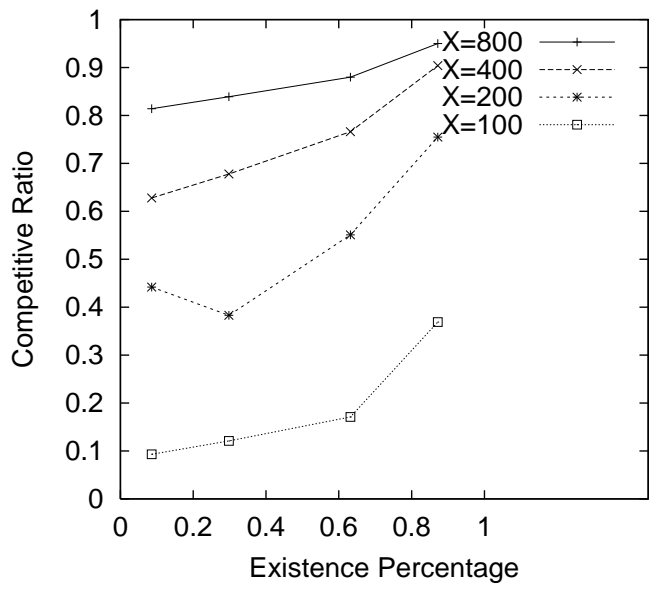

(a) Limited granularity heuristic

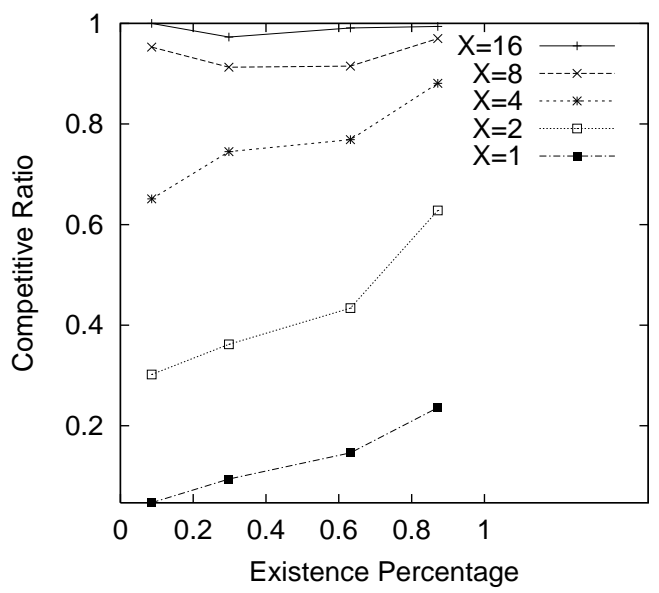

(b) Limited path heuristic

Figure 8: 2-constrained problems on $16 \times 16$ meshes

with the same QoS constraints on 500 randomly generated $8 \times 8$ meshes. In this experiment, finding paths with constraints $(47.5,95.0)$ results in an existence percentage of 0.170 . Constraints $(50.0,100.0)$ result in an existence percentage of 0.334 , constraints $(52.5,105.0)$ result in an existence percentage of 0.534 , constraints $(55.0,110.0)$ result in an existence percentage of 0.742 , and constraints $(57.5,115.0)$ result in an existence percentage of 0.866 . Notice that for experiments with meshes, the paths to be found are between the diagonal nodes in the network as shown in Figure 6 (a). The general trend is that both the limited granularity heuristics and the limited granularity heuristics can have close to 1 competitive ratio when a sufficiently large number of entries are maintained in each node. However, to achieve high competitive ratio, the limited granularity heuristic requires to maintain a very large number of entries, e.g. 800 in this experiment, while the limited path heuristic only requires a small number of en- 


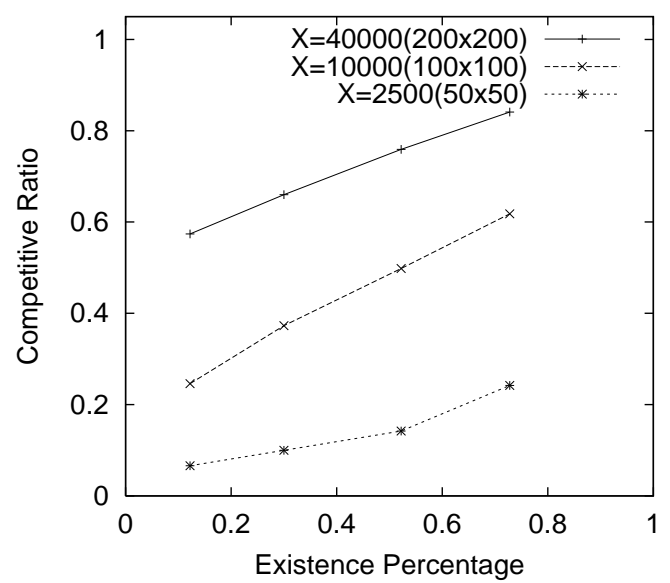

(a) Limited Granularity Heuristic

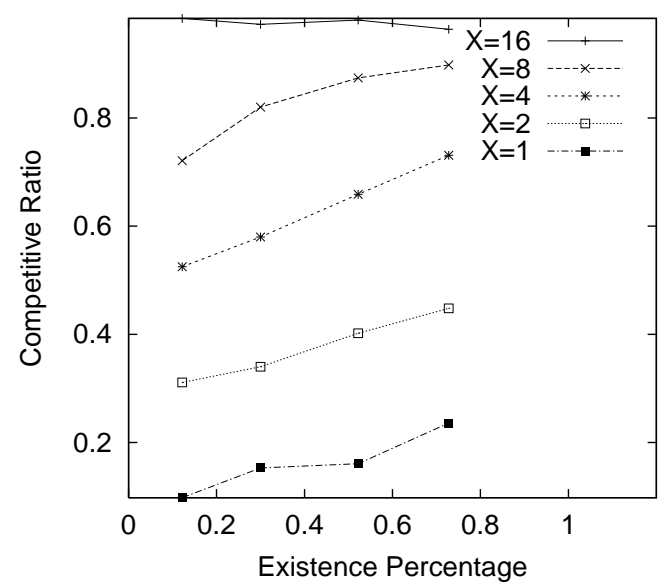

(b) Limited Path Heuristic

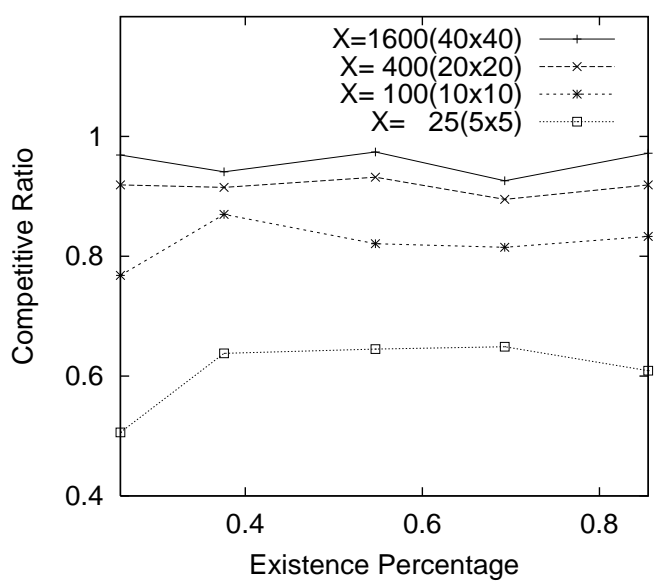

(a) Limited Granularity Heuristic

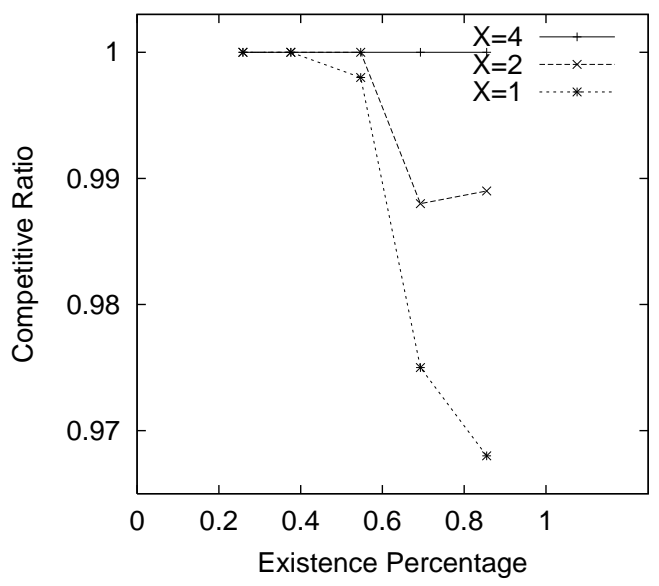

(b) Limited Path Heuristic
Figure 9: 3-constrained problems on $8 \times 8$ meshes

tries in each node, e.g. 8 in the experiment. Due to the large difference in the number of entries maintained in each node, the limited path heuristic is also much more efficient in terms of execution time than the limited granularity heuristic.

Figure 8 shows the performance of the heuristics for $16 \times$ 16 meshes with 2 QoS constraints. The data are obtained by running the two heuristics and the exhaustive algorithm using requests with the same QoS constraints on 500 randomly generated $16 \times 16$ meshes. In this experiment, finding paths with constraints $(95.0,190.0)$ results in an existence percentage of 0.086 . Constraints $(100.0,200.0)$ result in an existence percentage of 0.294 , constraints $(105.0,210.0)$ result in an existence percentage of 0.632 , and constraints $(110.0,220.0)$ result in an existence percentage of 0.872 . The general trend in the $16 \times 16$ mesh is similar to that in the $8 \times 8$ mesh except that maintaining same amount entries in the larger mesh results in lower performance. For example, in the $8 \times 8$ mesh, the limited granularity heuris-
Figure 10: 3-constrained problems on the MCI backbone

tics has about $95 \%$ competitive ratio when maintaining 800 entries in each node while in the $16 \times 16$ mesh, it can only achieve $81.6 \%$ competitive ratio when finding paths with constraint $(95.0,190.0)$ (existence percentage: 0.086). The degradation in performance for the limited path heuristic is not so severe as that for the limited granularity heuristic. Maintaining 16 entries in each node can still achieve a close to $100 \%$ competitive ratio in the $16 \times 16$ mesh.

Figure 9 shows the performance of the two heuristics when they solve 3 -constrained problems in $8 \times 8$ meshes. The existence percentage and the competitive ratio for each point in the figure are obtained by solving 500 QoS routing problems with the same QoS requirement. Constraints $(52.5,105.0,157.5)$ result in an existence percentage of 0.122 , constraints $(55.0,110.0,165.0)$ result in an existence percentage of 0.300 , constraints $(57.5,115.0,172.5)$ result in an existence percentage of 0.522 , constraints $(60.0,120.0,180.0)$ result in an existence percentage of 0.728 . 


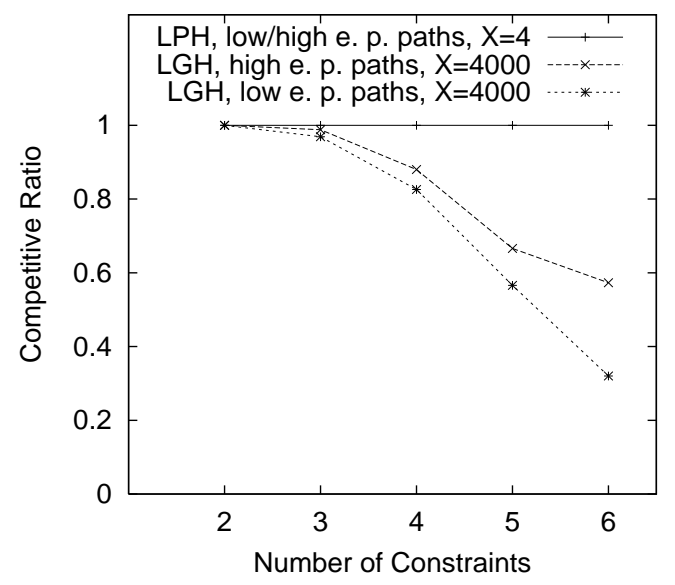

Figure 11: Impacts of the numbers of QoS constraints on the MCI backbone topology (LGH: limited granularity heuristic, LPH: limited path heuristic)

Comparing the results in Figure 9 and the results in Figure 7 , we can see that the number of entries to be maintained in each node for the limited granularity heuristic to be effective increases dramatically for 3-constrained problems comparing to 2-constrained problems. Maintaining a table of size 40000 (200x200) for 3-constrained problems yields worse competitive ratio than maintaining a table of size 200 for 2 -constrained problems. The competitive ratio of the limited path heuristic, on the other hand, only decreases slightly. Maintaining 16 entries result in more than $95 \%$ competitive ratio for all the cases in the experiments. This indicates that the limited path heuristic is much less sensitive to the number of QoS constraints than the limited granularity heuristic.

Figure 10 shows the results when the two heuristics solve 3 -constrained QoS routing problems in the MCI backbone topology. The existence percentage and the competitive ratio are obtained by solving 1000 QoS routing problems. Each of the 1000 routing problems tries to find a connection between the randomly generated source and destination with the same QoS requirement. In this experiment, constraints $(10.0,20.0,30.0)$ result in an existence percentage of 0.259 , constraints $(12.5,25.0,37.5)$ result in an existence percentage of 0.376 , constraints $(15.0,30.0,45.0)$ result in an existence percentage of 0.547 , constraints $(17.5,35.0,52.5)$ result in an existence percentage of 0.693 , constraints $(20.0,40.0,60.0)$ result in an existence percentage of 0.855 . The general trend in this figure is similar to that in the previous experiment. In comparison to the limited path heuristic, the limited granularity heuristic requires significantly more resources to achieve good performance. The limited granularity heuristic must maintain 1600 entries (a $40 \times 40$ table) in each node to consistently achieve $95 \%$ competitive ratio, while the limited path heuristic achieves close to $100 \%$ competitive ratio with 4 entries in each node.

Figure 11 shows the impact of the number of constraints on the performance of the heuristics using the MCI backbone topology. In this experiment, we fix the number of entries maintained at each node for both heuristics and study the performance of the two heuristics when they solve QoS routing problems with different numbers of QoS constraints. For the limited granularity heuristics, we fix the table size to be around 4,000. More specifically, we maintain in each node a linear array of 4,000 for 2-constrained problems, a $64 \times 64$ table for 3 -constrained problems, a $17 \times 17 \times 17$ table for 4 -constrained problems, a $8 \times 8 \times 8 \times 8$ table for 5 -constrained problems and a $6 \times 6 \times 6 \times 6 \times 6$ table for 6 -constrained problems. For the limited path heuristic, we fix the table size to be 4 . We consider two types of paths: high existence percentage paths and low existence percentage paths. The high existence percentage paths are paths that satisfy constraints $(20.0,40.0)$ for 2 -constrained problems, $(20.0,40.0,60.0)$ for 3 -constrained problems, $(20.0,40.0,60.0,80.0)$ for 4-constrained problems, $(20.0,40.0,60.0,80.0,100.0)$ for 5 -constrained problems, and $(20.0,40.0,60.0,80.0,100.0,120.0)$ for 6 -constrained problems. The existence percentage for these paths are between 0.75 and 0.95 . The low existence percentage paths are paths that satisfy constraints $(10.0,20.0)$ for 2 -constrained problems, $(10.0,20.0,30.0)$ for 3 -constrained problems, $(10.0,20.0,30.0,40.0)$ for 4 -constrained problems,

$(10.0,20.0,30.0,40.0,50.0)$ for 5 -constrained problems, and $(10.0,20.0,30.0,40.0,50.0,60.0)$ for 6 -constrained problems. The existence percentage for these paths are between 0.22 and 0.33 . The results are obtained by solving 1000 QoS routing problems for each setting. As can be seen from the figure, the performance of the limited path heuristic is somewhat insensitive to the number of QoS constraints. With $X=4$, the limited path heuristic achieves close to $100 \%$ competitive ratio for all different number of constraints. The performance of the limited granularity heuristic drastically degrades as the number of QoS constraints increases. The competitive ratio falls from $100 \%$ to $32 \%$ for low existence percentage paths and from $100 \%$ to $58 \%$ for high existence percentage paths when the number of constraints increases from 2 to 6 . This experiment confirms that the limited path heuristic is more efficient than the limited granularity heuristic in solving general $k-$ constrained problems when $k>3$.

\section{Conclusion}

In this paper, we study two heuristics, the limited granularity heuristic and the limited path heuristic, that can be applied to the extended Bellman-Ford algorithm to solve $k$-constrained QoS path routing problems. We show that although both heuristics can solve $k$-constrained QoS routing problems with high probability in polynomial time, to achieve high performance, the limited granularity heuristic requires much more resources than the limited path heuristic does. Specifically, the limited granularity heuristics must maintain a table of size $O\left(|N|^{k-1}\right)$ in each node to achieve good performance, which results in a time complexity of $O\left(|N|^{k}|E|\right)$, while the limited path heuristic only needs to maintain $O\left(|N|^{2} l g(|N|)\right)$ entries in each node. 
Both our analytical and simulation results indicate that the limited path heuristic is more efficient than the limited granularity heuristic in solving general $k$-constrained QoS routing problems when $k>3$, although previous research results show that both the limited granularity heuristic and the limited path heuristic can solve 2-constrained QoS routing problems efficiently. The advantage of the limited granularity heuristic, however, is that by maintaining a table of size $n^{k-1} N^{k-1}$ it guarantees finding $\left(1-\frac{1}{n}\right)$ approximate solutions while the limited path heuristic cannot provide such guarantee.

\section{Acknowledgment}

This work was supported in part by NSF CCR-9904943, CCR-0073482 and ANI-0106706. The author would like to thank Xingming Liu for his help in generating some simulation results and the anonymous referees for their valuable comments.

\section{References}

[1] S. Chen and K. Nahrstedt "On Finding Multi-Constrained Paths." IEEE International Conference on Communications (ICC'98), pp 874-879, June 1998.

[2] Shigang Chen and Klara Nahrstedt " An Overview of Qualityof-Service Routing for the Next Generation High-Speed Networks: Problems and Solutions," IEEE Network Magazine, Special Issue on Transmission and Distribution of Digital Video, Vol. 12, No. 6, pages 64-79, November-December 1998.

[3] T. H. Cormen, C. E. Leiserson and R. L. Rivest, "Introduction to Algorithms.", The MIT Press, 1990.

[4] J.M. Jaffe "Algorithms for Finding Paths with Multiple Constraints." Networks, Vol. 14, pp 95-116, 1984.

[5] Turgay Korkmaz, Marwan Krunz, and Spyros Tragoudas, "An efficient algorithm for finding a path subject to two additive constraints," ACM SIGMETRICS 2000 Conference, vol. 1, pp. 318-327, Santa Clara, CA, June 2000.

[6] Q. Ma and P. Steenkiste "Quality-of-Service Routing with Performance Guarantees", IFIP International Workshop on Quality of Service (IwQoS), pp 115-126, May 1997.

[7] Ariel Orda, "Routing with End-to-End QoS Guarantees in Broadband Networks", IEEE/ACM Transactions on Networking, Vol. 7, No. 3, pp 365-374, June 1999.

[8] H. F. Salama, D. S. Reeves and Y. Viniotis, "A Distributed Algorithm for Delay-Constrained Unicast Routing." IEEE INFOCOM, pp 84-91, April 1997.

[9] Z. Wang and J. Crowcroft "QoS Routing for Supporting Resource Reservation." IEEE Journal on Selected Areas in Communications, Vol. 14, No. 7, pp 1228-1234, Sept. 1996.

[10] R. Widyono, "The Design and Evaluation of Routing Algorithms for Real-time Channels." technical report TR-94-024, International Computer Science Institute, University of California at Berkeley, 1994.

\section{Appendix}

Lemma 3: For $m \geq 2, A_{2}^{m}(|S|, 0) \leq \frac{(2 \ln (|S|))^{m-1}}{|S| *(m-1) !}$.

Proof: We will first prove the following formula that will be used in the proof of the lemma. For any $n>2$,

$$
\sum_{i=2}^{n-1} \frac{\ln (i)}{i} \leq \frac{2 \ln n^{m+1}(n)}{m+1}
$$

For $i \geq 2$ and $i+1>x \geq i, \frac{\ln ^{m}(i)}{i}<\frac{2 \ln ^{m}(x)}{x}$. Hence, $\sum_{i=2}^{n-1} \frac{\ln ^{m}(i)}{i} \leq \int_{2}^{n} \frac{2 \ln ^{m}(x)}{x} d x=\left.\frac{2}{m+1} \ln ^{m+1}(x)\right|_{2} ^{n} \leq \frac{2 l n^{m+1}(n)}{m+1}$

Armed with this formula, we will now prove the theorem:

$$
\begin{aligned}
& A_{2}^{m}(|S|, 0) \\
& =\frac{1}{|S|} \sum_{l_{1}=m-1}^{|S|-1} \frac{1}{l_{1}} \sum_{l_{2}=m-2}^{l_{1}-1} \frac{1}{l_{2}} \sum \ldots \sum_{l_{m-1}=1}^{l_{m-2}-1} \frac{1}{l_{m-1}} \\
& \leq \frac{1}{|S|} \sum_{l_{1}=m-1}^{|S|-1} \frac{1}{l_{1}} \sum_{l_{2}=m-2}^{l_{1}-1} \frac{1}{l_{2}} \sum \ldots \sum_{l_{m-2}=2}^{l_{m-3}-1} \frac{\ln \left(l_{m-2}\right)+1}{l_{m-2}} \\
& \leq \frac{1}{|S|} \sum_{l_{1}=m-1}^{|S|-1} \frac{1}{l_{1}} \sum_{l_{2}=m-2}^{l_{1}-1} \frac{1}{l_{2}} \sum \ldots \sum_{l_{m-2}=2}^{l_{m-3}-1} \frac{2 l n\left(l_{m-2}\right)}{l_{m-2}} \\
& \leq \frac{1}{|S|} \times \frac{2}{1 !} \sum_{l_{1}=m-1}^{|S|-1} \frac{1}{l_{1}} \sum_{l_{2}=m-2}^{l_{1}-1} \frac{1}{l_{2}} \sum \ldots \sum_{l_{m-2}=2}^{l_{l}-1} \frac{\ln \left(l_{m-2}\right)}{l_{m-2}} \\
& \leq \frac{1}{|S|} \times \frac{2^{2}}{2 !} \sum_{l_{1}=m-1}^{|S|-1} \frac{1}{l_{1}} \sum_{l_{2}=m-2}^{l_{1}-1} \frac{1}{l_{2}} \sum \ldots \sum_{l_{m-3}=3}^{l_{m-4}-1} \frac{\ln ^{2}\left(l_{m-3}\right)}{l_{m-3}} \\
& \leq \ldots \\
& \leq \frac{1}{|S|} \times \frac{2^{m-1}}{(m-1) !} \times(\ln (|S|))^{m-1}=\frac{(2 l g(|S|))^{m-1}}{|S| *(m-1) !}
\end{aligned}
$$

Lemma 5: $P_{k}^{i, j}>P_{k}^{i+1, j}$.

Proof: Base case, $k=2, P_{2}^{i, j}=\frac{1}{i}>\frac{1}{i+1}=P_{2}^{i+1, j}$. Induction case, assuming that for any $i, j$ and $k$, $P_{k}^{i, j}>P_{k}^{i+1, j}$,

$$
\begin{aligned}
P_{k+1}^{i, j} & =\frac{1}{i} \sum_{l=0}^{j} P_{k}^{i-l, j-l} \\
& >\frac{1}{i+1} \sum_{l=0}^{j} P_{k}^{i+1-l, j-l} \\
& =P_{k+1}^{i+1, j} \square
\end{aligned}
$$

Lemma 6: For $k \geq 3$ and $0 \leq j \leq|S|$,

$$
\sum_{i=0}^{|S|} A_{k}(i, j)=\sum_{i=j+1}^{|S|} P_{k}^{i, j}<2 .
$$

Proof: Base case, $k=3$. From Lemma 4 , we obtain

$$
P_{3}^{i, j}=\frac{1}{i}\left(\sum_{l=0}^{j} P_{2}^{i-l, j-l}\right)=\frac{1}{i}\left(\frac{1}{i}+\frac{1}{i-1}+\ldots+\frac{1}{i-j}\right) .
$$

For any $j$,

$$
\begin{aligned}
& \sum_{i=0}^{|S|} A_{3}(i, j)-\sum_{i=0}^{|S|} A_{3}(i, j+1) \\
& =\sum_{i=j+1}^{|S|} P_{3}^{i, j}-\sum_{i=j+2}^{|S|} P_{3}^{i, j+1} \\
& =\frac{1}{j}\left(\frac{1}{j}+\frac{1}{j-1}+\ldots+\frac{1}{1}\right)-\left(\frac{1}{j+1} \frac{1}{1}+\frac{1}{j+2} \frac{1}{2}+\ldots+\frac{1}{i} \frac{1}{i-j-1}\right) \\
& >0
\end{aligned}
$$

Thus,

$$
\begin{aligned}
2 & >\sum_{i=1}^{|S|} \frac{1}{i^{2}} \\
& =\sum_{i=0}^{|S|} A_{3}(i, 0) \\
& >\sum_{i=0}^{|\bar{S}|} A_{3}(i, 1) \\
& >\ldots \\
& >\sum_{i=0}^{|S|} A_{3}(i,|S|) .
\end{aligned}
$$

Induction case, for any $j$ and $k$, assume

$$
\begin{aligned}
\sum_{i=0}^{|S|} A_{k}(i, j)=\sum_{i=j+1}^{|S|} P_{k}^{i, j}<2 . & \\
\sum_{i=0}^{|S|} A_{k+1}(i, j) & =\sum_{i=j+1}^{|S|} P_{k+1}^{i, j} \\
& =\sum_{i=j+1}^{|S|} \frac{1}{i} \sum_{l=0}^{j} P_{k}^{i-l, j-l} \\
& <\frac{1}{j+1} \sum_{l=0}^{j} \sum_{i=l+1}^{|S|} P_{k}^{i, l} \\
& \leq \frac{1}{j+1} *(2 *(j+1))=2
\end{aligned}
$$

Lemma 7: $P_{k}^{i, j} \leq \frac{1}{i}\left(\frac{1}{i}+\frac{1}{i-1}+\ldots+\frac{1}{i-j}\right)^{k-2}$.

Proof: Base case, $k=2$,

$$
P_{2}^{i, j}=\frac{1}{i} \leq \frac{1}{i}\left(\frac{1}{i}+\frac{1}{i-1}+\ldots+\frac{1}{i-j}\right)^{2-2} .
$$

Induction case, assume that for any $i, j$ and $k$,

$$
P_{k}^{i, j} \leq \frac{1}{i}\left(\frac{1}{i}+\frac{1}{i-1}+\ldots+\frac{1}{i-j}\right)^{k-2} .
$$

$$
\begin{aligned}
& P_{k+1}^{i, j} \\
& =\frac{1}{i}\left(P_{k}^{i, j}+P_{k}^{i-1, j-1}+\ldots+P_{k}^{i-j, 0}\right) \\
& \leq \frac{1}{i}\left(\frac{1}{i}\left(\frac{1}{i}+\frac{1}{i-1}+\ldots+\frac{1}{i-j}\right)^{k-2}\right. \\
& \left.\quad+\frac{1}{i-1}\left(\frac{1}{i-1}+\frac{1}{i-2} \ldots+\frac{1}{i-j}\right)^{k-2}+\ldots+\frac{1}{i-j}\left(\frac{1}{i-j}\right)^{k-2}\right)
\end{aligned}
$$




$$
\begin{aligned}
\leq & \frac{1}{i}\left(\frac{1}{i}\left(\frac{1}{i}+\frac{1}{i-1}+\ldots+\frac{1}{i-j}\right)^{k-2}\right. \\
& +\frac{1}{i-1}\left(\frac{1}{i}+\frac{1}{i-1}+\ldots+\frac{1}{i-j}\right)^{k-2}+\ldots \\
& \left.+\frac{1}{i-j}\left(\frac{1}{i}+\frac{1}{i-1}+\ldots+\frac{1}{i-j}\right)^{k-2}\right) \\
= & \frac{1}{i}\left(\left(\frac{1}{i}+\ldots+\frac{1}{i-j}\right)\left(\frac{1}{i}+\ldots+\frac{1}{i-j}\right)^{k-2}\right) \\
= & \frac{1}{i}\left(\frac{1}{i}+\frac{1}{i-1}+\ldots+\frac{1}{i-j}\right)^{k-1}
\end{aligned}
$$

Lemma 8: For a constant $k$, there exists a constant $c$ such that $\sum_{i=1}^{\infty} \frac{1}{2^{i}} i^{k} \leq c$.

Proof: When $k=0, \sum_{i=1}^{\infty} \frac{1}{2^{i}} i^{k}=\sum_{i=1}^{\infty} \frac{1}{2^{i}}=1$.

Let $Y(k)=\sum_{i=1}^{\infty} \frac{1}{2^{i}} i^{k}, \frac{Y(k)}{2}=\sum_{i=2}^{\infty} \frac{x}{2^{i}}(i-1)^{k}$

$$
\begin{aligned}
\frac{Y(k)}{2} & =Y(k)-\frac{Y(k)}{2} \\
& =\frac{1}{2}+\sum_{i=2}^{\infty} \frac{1}{2^{i}}\left(i^{k}-(i-1)^{k}\right) \\
& \leq \frac{1}{2}+\sum_{i=2}^{\infty} \frac{1}{2^{i}}\left(k * i^{k-1}\right) \\
& \leq k * Y(k-1)
\end{aligned}
$$

Thus, $Y(k) \leq 2 k Y(k-1) \leq 2^{2} k(k-1) * Y(k-2) \leq \ldots \leq$ $2^{k} k ! Y(0)=2^{k} k !$. When $k$ is a constant, there exists a constant $c=2^{k} k$ ! such that $\sum_{i=1}^{\infty} \frac{1}{2^{i}} i^{k} \leq c$.

Lemma 9: For a constant $\mathrm{k}$ and $1 \leq j \leq i-1$, there exists a constant $c$ such that

$$
\sum_{n=j+1}^{i-1}\left(\frac{1}{i}+\frac{1}{i-1}+\ldots \frac{1}{i-n}\right)^{k}\left(\frac{1}{n}+\ldots+\frac{1}{n-j}\right)^{k} \leq c * i .
$$

Proof: Let $W(m)=\left(\frac{1}{i}+\frac{1}{i-1}+\ldots+\frac{1}{i-m}\right)^{k}$. We will first derive some bounds for $W(m)$.

For $1 \leq m \leq \frac{i}{2}$,

$$
\begin{aligned}
W(m) & =\left(\frac{1}{i}+\frac{1}{i-1}+\ldots+\frac{1}{i-m}\right)^{k} \\
& \leq\left(\frac{1}{i / 2}+\frac{1}{i / 2}+\ldots+\frac{1}{i / 2}\right)^{k} \\
& \leq\left(m * \frac{1}{i / 2}\right)^{k} \leq 1^{k} .
\end{aligned}
$$

For $\frac{i}{2}+1 \leq m \leq \frac{3 i}{4}$,

$$
W(m) \leq\left(\frac{1}{i / 2}+\frac{1}{i / 2}+\ldots+\frac{1}{i / 2}+\left(m-\frac{i}{2}\right) * \frac{1}{i / 4}\right)^{k} \leq 2^{k} .
$$

In general, for $\frac{\left(2^{j}-1\right)}{2^{j}} * i+1 \leq m \leq \frac{\left(2^{j+1}\right)-1}{2^{j+1}} * i$,

For $n \leq i$, we also have

$$
W(m) \leq(j+1)^{k} \text {. }
$$

$$
\left(\frac{1}{n}+\ldots+\frac{1}{n-j}\right)^{k} \leq\left(\frac{1}{i}+\ldots+\frac{1}{n-j}\right)^{k}=W(i-n+j) .
$$

Thus,

$$
\begin{aligned}
& \sum_{n=j+1}^{i-1}\left(\frac{1}{i}+\frac{1}{i-1}+\ldots \frac{1}{i-n}\right)^{k}\left(\frac{1}{n}+\ldots+\frac{1}{n-j}\right)^{k} \\
& \leq \sum_{n=j+1}^{i-1} W(n) W(i-n+j) \\
& =W(i-1) W(j+1)+\ldots+W(j+1) W(i-1) \\
& \leq W(j+1)^{2}+\ldots+W(i-1)^{2} / * a^{2}+b^{2} \geq 2 a b^{*} / \\
& =\sum_{n=j+1}^{i-1}(W(n))^{2} \leq \sum_{n=1}^{i-1}(W(n))^{2} \\
& =\sum_{n=1}^{\frac{i}{2}}(W(n))^{2}+\sum_{n=\frac{i}{2}+1}^{\frac{3 i}{4}}(W(n))^{2}+\ldots \\
& =\frac{i}{2} 1^{2 k}+\frac{i}{4} 2^{2 k}+\frac{i}{8} 3^{2 k}+\ldots
\end{aligned}
$$

$\leq c * i$, where $c$ is a constant. $/^{*}$ Applying Lemma $8 * / \square$

Lemma 10: Let $0 \leq j<\frac{i}{2}$, there exists a constant $c$ such that

$$
\sum_{n=j+1}^{i-1} P_{k}^{n, j}\left(\frac{1}{i}+\ldots+\frac{1}{i-n}\right)^{m} \leq c .
$$

Proof: From Lemma 7, we have $\sum_{n=j+1}^{i-1} P_{k}^{n, j}<2$. From Lemma 6 , we have $P_{k}^{i, j}>P_{k}^{i+1, j}$. Hence,

$$
\begin{aligned}
& \sum_{n=j+1}^{\frac{i}{2}} P_{k}^{n, j}<2 \\
& \sum_{n=\frac{i}{2}+1}^{\frac{3 i}{4}} P_{k}^{n, j}<2 \\
& \sum_{n=\frac{3 i}{4}+1}^{\frac{7 i}{8}} P_{k}^{n, j}<\frac{1}{2} \sum_{n=\frac{i}{2}+1}^{\frac{3 i}{4}} P_{k}^{n, j}<2 * \frac{1}{2} \\
& \sum_{n=\frac{7 i}{8}+1}^{\frac{15 i}{16}} P_{k}^{n, j}<\frac{1}{4} \sum_{n=\frac{i}{2}+1}^{\frac{3 i}{4}} P_{k}^{n, j}<2 * \frac{1}{4}
\end{aligned}
$$$$
\text { ... }
$$

Thus,

$$
\begin{aligned}
& \sum_{n=j+1}^{i-1} P_{k}^{n, j}\left(\frac{1}{i}+\ldots+\frac{1}{i-n}\right)^{m} \\
& =\sum_{n=j+1}^{\frac{i}{2}} P_{k}^{n, j}\left(\frac{1}{i}+\ldots+\frac{1}{i-n}\right)^{m} \\
& \quad+\sum_{n=\frac{3 i}{4}}^{\frac{3 i}{2}} P_{k}^{n, j}\left(\frac{1}{i}+\ldots+\frac{1}{i-n}\right)^{m} \\
& \quad+\sum_{n=\frac{3 i}{4}+1}^{\frac{7 i}{8}} P_{k}^{n, j}\left(\frac{1}{i}+\ldots+\frac{1}{i-n}\right)^{m}+\ldots \\
& \leq 1^{m} \sum_{n=\frac{3 i}{4}}^{\frac{3 i}{4}} P_{k}^{n, j}+2^{m} \sum_{n=\frac{i}{2}+1}^{\frac{3 i}{4}} P_{k}^{n, j} \\
& \quad+3^{m} \sum_{n=\frac{3 i}{4}+1}^{\frac{7 i}{8}} P_{k}^{n, j}+\ldots \\
& \leq 1^{m} * 2+2\left(2^{m} * \frac{1}{2^{0}}+3^{m} * \frac{1}{2^{1}}+4^{m} * \frac{1}{2^{2}}+\ldots\right) \\
& \leq c, \text { where } c \text { is a constant. } /^{*} \text { applying Lemma } 8 * /
\end{aligned}
$$

Xin Yuan Xin Yuan (M '98 / ACM' 98) received his PH.D degree in Computer Science from the University of Pittsburgh. He is an assistant professor at the department of Computer Science, Florida State University. His research interests include quality of service routing, optical WDM networks and high performance communication for clusters of workstations. His email address is: xyuan@cs.fsu.edu. 